\title{
A REMARK ON HOLOMORPHIC SECTIONS OF CERTAIN HOLOMORPHIC FAMILIES OF RIEMANN SURFACES
}

\author{
YOICHI IMAYOSHI AND TOSHIHIRO NOGI
}

\begin{abstract}
In this paper, we study a special holomorphic family $(M, \pi, R)$ of closed Riemann surfaces of genus two over a fourth punctured torus $R$, which is a kind of a Kodaira surface and is constructed by Riera. We give two explicit defining equations for $(M, \pi, R)$ by using elliptic functions, and determine all the holomorphic sections of $(M, \pi, R)$. Proofs will appear elsewhere.
\end{abstract}

\section{INTRODUCTION}

Let us consider a Diophantine equation

$$
\sum_{i+j+k=N} A_{i j k} X^{i} Y^{j} Z^{k}=0
$$

over the function field $K$ of a closed Riemann surgace $\hat{R}$. Here $K$ is the field of all meromorphic functions on $\hat{R}$ and the coefficients $A_{i j k}$ are elements of $K$. The problem is to find the solutions $(X, Y, K) \in P^{2}(K)$ of the function equation (1) over $\hat{R}$.

This problem is reformulated geometrically in the following way. It is assumed that we find a Zariski open subset $R$ of $\hat{R}$ and a Zariski open subset $M$ of the algebraic surface $\hat{M}$ defined by

$$
\hat{M}=\left\{([x, y, z], t) \in P^{2}(\mathbf{C}) \times \hat{R} \mid \sum_{i+j+k=N} A_{i j k}(t) x^{i} y^{j} z^{k}=0\right\}
$$

such that the holomorphic map $\pi: M \rightarrow R$ given by $\pi([x, y, z], t)=t$ satisfies the two conditions:

(1) $\pi$ is of maximal rank at every point of $M$, and

(2) for every $t \in R$, the fiber $S_{t}=\pi^{-1}(t)$ of $M$ over $t$ is a Riemann surface of fixed finite type $(g, n)$, where $g$ is the genus of $S_{t}$ and $n$ is the number of punctures of $S_{t}$.

We call such a triplet $(M, \pi, R)$ is a holomorphic family of Riemann surfaces of type $(g, n)$ over $R$.

We assume throughout this paper that a holomorphic family $(M, \pi, R)$ of Riemann surfaces is of type $(g, n)$ with $2 g-2+n>0$ and the base surface $R$ is of finte type, i.e., a Riemann surface obtained by removing at most a finite number of points from a closed Riemann surface.

1991 Mathematics Subject Classification. Primary 11G30, Secondary 14H15, 32G15, 32J25.

Key words and phrases. holomprhoc section, holomorphic family of Riemann surfaces, function field, Kodaira surface, moduli space, Teichmüller space, Diophantine equation. 
Two holomorphic families $\left(M_{1}, \pi_{1}, R\right)$ and $\left(M_{2}, \pi_{2}, R\right)$ of Riemann surfaces are called isomorphic if there exists a biholomorphic map $f: M_{1} \rightarrow M_{2}$ with $\pi_{1}=\pi_{2} \circ f$. A holomorphic family $(M, \pi, R)$ of Riemann surfaces is locally trivial if for every point $p_{0} \in R$ there exsits a neighborhood $U_{0}$ of $p_{0}$ in $R$ such that $\left(\pi^{-1}\left(U_{0}\right), \pi \mid \pi^{-1}\left(U_{0}\right), U_{0}\right)$ is isomorphic to the trivial holomorphic family $\left(U_{0} \times S_{p_{0}}, \pi_{0}, U_{0}\right)$, where $\pi_{0}: U_{0} \times S_{p_{0}} \rightarrow U_{0}$ is the canonical projection. It is known that a holomorphic family $(M, \pi, R)$ of Riemann surfaces is locally trivial if and only if the fibers $S_{t}$ are all isomorphic.

A holomorphic map $s: R \rightarrow M$ is said to be a holomorphic section of a holomorphic family $(M, \pi, R)$ of Riemann surfaces if s stisfies $\pi \circ s=$ id on $R$. Note that if $(X, Y, K) \in$ $P^{2}(K)$ satisfies the function equation $(1)$, then $s(t)=([X(t), Y[t], Z[t], t)$ gives rise to a holomorphic section of $(M, \pi, R)$.

By using theory of Teichmüller space, Imayoshi and Shiga in [6] gave a proof for a finiteness theorem of sections (Mordell conjecture) and a finiteness theorem of families (Shafarevich conjecture). See also Arakelov [1], Faltings [3], Grauert [4], Jost and Yau [7], Manin [9], McMullen [10], and Parshin [11].

In this paper we deal with a special holomorphic family $(M, \pi, R)$ of closed Riemann surfaces of genus two over a fourth punctured torus $R$, which is a kind of a Kodaira surface as [8] and is constructed by Riera in [12]. We give two explicit defining equations for $(M, \pi, R)$ by using elliptic functions, and determine all the holomorphic sections of $(M, \pi, R)$.

\section{Construction of a Certain holomorphic family $(M, \pi, R)$ of Riemann SURFACES}

Now we explain briefly a construction of our holomorphic family $(M, \pi, R)$ of Riemann surfaces, which is due to Riera [12].

Take a point $\tau$ in the upper half-plane $\mathbf{H}$ in the complex plane $\mathbf{C}$. Let $\Gamma_{1, \tau}$ be the discrete subgroup of $\operatorname{Aut}(\mathbf{C})$ generated by two translations $z \mapsto z+1$ and $z \mapsto z+\tau$. Denote by $\hat{T}$ a torus defiend by the quotient space $\mathbf{C} / \Gamma_{1, \tau}=\{[z] \mid z \in \mathbf{C}\}$. We set $p_{0}=[0] \in \hat{T}$ and $T=\hat{T} \backslash\left\{p_{0}\right\}$.

For a point $p \in T$ we take two replicas of the torus $\hat{T}$ cut along a simple arc from $p$ to $p_{0}$, and call them sheet I and sheet II. The cut on each sheet has two edges, labeled + edge and - edge. To construct a Riemann surface $X_{p}$, we attach the + edge on sheet I and the - edge on sheet II, and then attach the + edge on sheet II and the - edge on sheet I. Then we obtain a closed Riemann surface $X_{p}$ of genus two and the two-sheeted covering $X_{p} \rightarrow \hat{T}$ which is branched over $p_{0}$ and $p$ with branch order 2 . It should be noted that the above procedure depends, of course, not only on the choice of the point $p$ but also on the choice of the "cut" from $p$ to $p_{0}$. Essentially we can take four different "cuts" $\alpha_{1}, \alpha_{2}, \alpha_{3}$, and $\alpha_{4}$ between $p$ and $p_{0}$ (see Fig.1).

To specify the "cut" we construct a four-sheeted unbranched covering

$$
\rho: R \rightarrow T
$$

of $T$ such that $R$ is a torus with four punctures as follows: Let $\Gamma_{2,2 \tau}$ be the discrete subgroup of $\operatorname{Aut}(\mathbf{C})$ generated by two translations $z \mapsto z+2$ and $z \mapsto z+2 \tau$. Denote by $\hat{R}$ a torus defiend by the quotient space $\mathbf{C} / \Gamma_{2,2 \tau}=\{[z] \mid z \in \mathbf{C}\}$. Let $\hat{\rho}: \hat{R} \rightarrow \hat{T}$ be the canonical projection given by $\hat{\rho}([z])=[z]$. We set $R=\hat{\rho}^{-1}(T)$ and $\rho=\hat{\rho} \mid R$. The good thing is that a point $t=[z] \in R$ determines a point $p=\rho([z]) \in T$ and a "cut" 
$\alpha=\hat{\rho}(\beta)$ from $p$ to $p_{0}=[0]$, where $\beta$ is a simple arc on $\hat{R}$ from [0] to $t$. Denote by $S_{t}$ the closed Riemann surface of genus two which is a two-sheeted branched covering surface of $\hat{T}$ constructed by a "cut" $\alpha=\hat{\rho}(\beta)$. Note that the two-sheeted branched covering $\Pi_{t}: S_{t} \rightarrow \hat{T}$ is uniquely determined by the choice of $t \in R$ and does not depend on $\beta$.
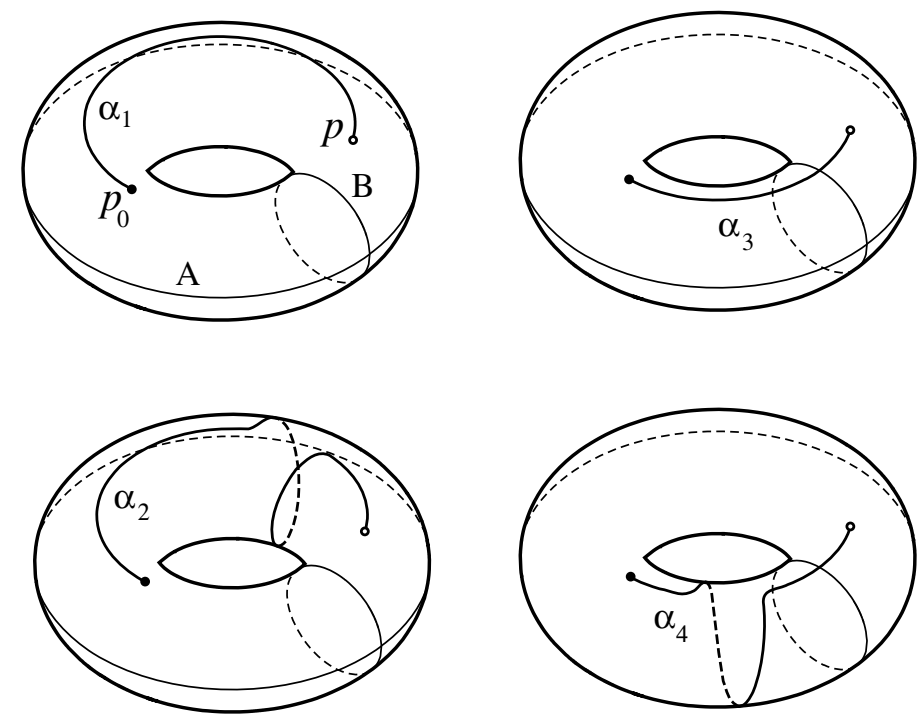

FIGURE 1. For each $p \in T$, there are precisely four two-sheeted branched coverings $S_{t_{i}} \rightarrow \hat{T}$, where $t_{i} \in R$ with $\hat{\rho}\left(t_{i}\right)=p$ and the cut $\alpha_{i}$ is given by the projection $\hat{\rho}\left(\beta_{i}\right)$ of a simple arc $\beta_{i}$ between [0] and $t_{i}$ on $\hat{R}$.

Now we have the following theorem (see Riera [12]):

Theorem 1. In the above situation, let

$$
\begin{aligned}
& M=\bigsqcup_{t \in R}\{t\} \times S_{t}, \\
& \pi: M \rightarrow R, \pi(t, q)=t .
\end{aligned}
$$

Then $(M, \pi, R)$ is a holomorphic family of closed Riemann surfaces of genus two over a fourth punctured torus $R$.

\section{Defining equations for $(M, \pi, R)$}

In this section we find defining equations for $(M, \pi, R)$. For any point $t=[\tilde{t}] \in R$, Abel's theorem shows there exists a meromorphic function $f_{t}$ on $\hat{T}$ which has two zeros [0] and $\rho(t)$ of order one, and a pole $q_{t}=\rho(t) / 2$ of order two. Moreover in order to determine $f_{t}$ uniquely, we assume that $\left(d f_{t} / d z\right)([0])=1$. This function $f_{t}$ is given explicitly as follows (see Clemens [2]): 


$$
\begin{aligned}
f_{t}([z])= & \frac{1}{\theta^{\prime}(1 / 2+\tau / 2)} \times \frac{\theta(-\tilde{t} / 2+1 / 2+\tau / 2)^{2}}{\theta(-\tilde{t}+1 / 2+\tau / 2)} \times \\
& \frac{\theta(z+1 / 2+\tau / 2) \theta(z-\tilde{t}+1 / 2+\tau / 2)}{\theta(z-\tilde{t} / 2+1 / 2+\tau / 2)^{2}} .
\end{aligned}
$$

Here the theta function $\theta(z, \tau)$ is defined by

$$
\theta(z, \tau)=\sum_{k=-\infty}^{\infty} e^{\pi i\left(k^{2} \tau+2 k z\right)}, \quad z \in \mathbf{C} .
$$

Then we have the following assertion:

Theorem 2. In the above situation, let

$$
\begin{aligned}
& M_{E}=\left\{(t, p, w) \in R \times \hat{T} \times \hat{\mathbf{C}} \mid w^{2}=f_{t}(p)\right\}, \\
& \pi_{E}: M_{E} \rightarrow R, \pi_{E}(t, p, w)=t .
\end{aligned}
$$

Then the triplet $\left(M_{E}, \pi_{E}, R\right)$ is a holomorphic family of closed Riemann surfaces of genus two, and it is isomorphic to $(\mathcal{M}, \pi, R)$ in Theorem 1.

We find another defining equation for $(M, \pi, R)$. The holomorophic map $f_{t}: \hat{T} \rightarrow \hat{\mathbf{C}}$ has four branch points $q_{t}$ (pole), $a(t), b(t)$, and $c(t)$, where

$$
\begin{aligned}
& a(t)=f_{t}([(\tilde{t}+1) / 2]), \\
& b(t)=f_{t}([(\tilde{t}+\tau) / 2]), \\
& c(t)=f_{t}([(\tilde{t}+1+\tau) / 2]) .
\end{aligned}
$$

Let $g_{t}$ be the meromorphic function on $\hat{T}$ of degree 3 satisfying

(1) $g_{t}$ has simple zeros $[(\tilde{t}+1) / 2],[(\tilde{t}+\tau) / 2],[(\tilde{t}+1+\tau) / 2]$,

(2) $g_{t}$ has a pole $[\tilde{t}]$ of order 3 , and

(3) $g_{t}([0])=i$.

This function $g_{t}$ is given by

$$
\begin{aligned}
g_{t}(z)=i e^{-2 \pi i z} \times & \frac{\theta(-\tilde{t} / 2+1 / 2+\tau / 2)^{3}}{\theta(-\tilde{t} / 2) \theta(-\tilde{t} / 2+1 / 2) \theta(-\tilde{t} / 2+\tau / 2)} \times \\
& \frac{\theta(z-\tilde{t} / 2) \theta(z-\tilde{t} / 2+1 / 2) \theta(z-\tilde{t} / 2+\tau / 2)}{\theta(z-\tilde{t} / 2+1 / 2+\tau / 2)^{3}} .
\end{aligned}
$$

Setting $x=f_{t}, y=g_{t}$, we have a functional relation

$$
y^{2}=\frac{1}{a(t) b(t) c(t)}(x-a(t))(x-b(t))(x-c(t))
$$

on $\hat{T}$.

Now we have the following theorem: 
Theorem 3. In the above situation, let

$$
\begin{aligned}
P_{t}(x) & =\left(x^{2}-a(t)\right)\left(x^{2}-b(t)\right)\left(x^{2}-c(t)\right), \\
M_{H E} & =\left\{(t, x, y) \in R \times \hat{\mathbf{C}} \times \hat{\mathbf{C}} \mid y^{2}=P_{t}(x)\right\}, \\
\pi_{H E}: & M_{H E} \rightarrow R, \pi_{H E}(t, x, y)=t .
\end{aligned}
$$

Then the triplet $\left(M_{H E}, \pi_{H E}, R\right)$ is a holomorphic family of closed Riemann surfaces of genus two, and it is isomorphic to $(M, \pi, R)$ in Theorem 1.

\section{Holomorphei SECtions $(M, \pi, R)$}

Let us study the holomorphic sections of $(M, \pi, R)$ in Theorem 1 . The following is our main theorem in this paper.

Theorem 4. The holomorphic family $(M, \pi, R)$ of closed Riemann surfaces of genus two in Theorem 1 has exactly two holomorphic sections $s_{1}, s_{2}$. These sections are given by $s_{1}(t)=\left(t, p_{0}\right)$ and $s_{2}(t)=(t, \rho(t))$ for every $t \in R$.

In order to prove this theorem, we need the following two theorems (cf. Imayoshi [5], Theorem 4 and Theorem 5):

Theorem 5. The holomorphic family $(M, \pi, R)$ in Theorem 1 has a canonical completion $(\hat{M}, \hat{\pi}, \hat{R})$, where $\hat{M}$ is a compact two dimensional normal complex analytic space and $\hat{\pi}: \hat{M} \rightarrow \hat{R}$ is holomorphic. Moreover every holomorphic section $s: R \rightarrow M$ has a holomorphic extension $\hat{s}: \hat{R} \rightarrow \hat{M}$.

Theorem 6. The holomorphic map $\Pi: M=\bigsqcup_{t \in R}\{t\} \times S_{t} \rightarrow \hat{T}$ defined by $\Pi(t, q)=$ $\Pi_{t}(q)$ has a holomorphic extension $\hat{\Pi}: \hat{M} \rightarrow \hat{T}$.

Theorem 6 is proved by Theorems 2,3 , and 5 .

Now we can prove Theorem 4 as follows: Let $s: R \rightarrow M$ be an arbitrary holomorphic section of $(M, \pi, R)$. Theorem 5 and 6 imply that the holomorphic map $\varphi=\Pi \circ s: R \rightarrow \hat{T}$ has a holomorphic extention $\hat{\varphi}=\hat{\Pi} \circ \hat{s}: \hat{R} \rightarrow \hat{T}$. Let $\tilde{\varphi}: \mathbf{C} \rightarrow \mathbf{C}$ is a lift of $\hat{\varphi}: \hat{R} \rightarrow \hat{T}$. Then $\tilde{\varphi}(z)=A z+B, z \in \mathbf{C}$ for some constants $A, B \in \mathbf{C}$. Since $\varphi=\Pi \circ s$, we can show that we may assume that $A=0, B=0$, or $A=1, B=0$. In the case $A=0, B=0$, we have the section $s_{1}(t)=\left(t, p_{0}\right)$, and in the case $A=1, B=0$, we have the section $s_{2}(t)=(t, \rho[t])$.

\section{REFERENCES}

[1] S. Arakelov, Families of algebraic curves with fixed degeneracies, Math. USSR Izv. 35 (1971), 12691293.

[2] C.H. Clemens, A Scrapbook of Complex Curve Theory, Plenum Press, New York and London, 1980.

[3] G. Faltings, Endlichkeitssätze für abelsche Varietäten über Zahlkörpern, Invent. Math. 73(1983), 349-366.

[4] H. Grauert, Mordells Vermutung über rationale Punkte auf algebraischen Kurven und Funktionenkörper, IHES Publ. Math. 25(1965), 131-149.

[5] Y. Imayoshi, Holomorphic families of Riemann surfaces and Teichmüller spaces, in Riemann Surfaces and Related Topics, 1978 Stony Brook Conference, Ann. Math. Studies, No. 97, pp.277-300, edited by I. Kra and B. Maskit, Princeton University Press, Princeton, New Jersey, 1981.

[6] Y. Imayoshi and H. Shiga, A finiteness theorem of holomorphic families of Riemann surfaces, in Holomorphic Functions and Moduli, Vol. II, pp.207-219, edited by D. Drain et al, Springer-Verlag:MSRI Publications Vol.10, 1988. 
[7] J. Jost and S-T. Yau, Harmonic mappings and algebraic varieties over function fields, Amer. J. of Math. 115 (1993), 1197-1227.

[8] K. Kodaira, A certain type of irregular algebraic surfaces, J. d'Analyse Math. 19 (1967), 207-215.

[9] Y. Manin, A proof of the analog of the Mordell conjecture for algebraic curves over function fields, Soviet Math. Dokl. 152(1963),1061-1063.

[10] C. McMullen, From dynamics on surfaces to rational points on curves, Bull. Amer. Math. Soc. 37 (2000), 119-140.

[11] A.N. Parshin, Albegraic curves over function fields, Soviet Math. Dokl. 183(1968),524-526.

[12] G. Riera, Semi-direct products of Fuchsian groups and uniformization, Duke Math. J. 44 (1977), 291-304.

(Yoichi Imayoshi) Department of Mathematics, Osaka City University, Sugimoto, SumiyoshiKU, OSAKa 558-8585, JAPAN

E-mail address: imayoshi@sci.osaka-cu.ac.jp

(Toshihiro Nogi) Department of Mathematics, Osaka City University, Sugimoto, SumiyoshiKU, OSAKA 558-8585, JAPAN

E-mail address: nogicchi@ezweb.ne.jp 